\title{
Somatic loss of an EXT2 gene mutation during malignant progression in a patient with hereditary multiple osteochondromas
}

\author{
Nicolò Musso ${ }^{a, e, 1}$, Francesco Paolo Caronia ${ }^{b, 1}$, Sergio Castorina ${ }^{\mathrm{b}, \mathrm{c}}$, \\ Attilio Ignazio Lo Monte ${ }^{d}$, Vincenza Barresi ${ }^{a, e}$, Daniele Filippo Condorelli ${ }^{a, e, *}$ \\ a Scuola Superiore di Catania, University of Catania, Catania, Italy; ' Dipartimento di Chirurgia Toracica, Centro Clinico e \\ Diagnostico "G.B Morgagni", Catania, Italy; ' Department of Biomedical and Biotechnological Sciences, Section of Human \\ Anatomy and Histology, University of Catania, Catania, Italy; ${ }^{\mathrm{d}}$ Department DICHIRONS, Universita degli Studi di Palermo,

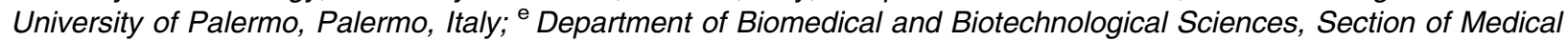 \\ Biochemistry, University of Catania, Catania, Italy
}

\begin{abstract}
Multiple osteochondromas (MO) is an autosomal-dominant skeletal disorder caused by mutations in the exostosin-1 (EXT1) or exostosin-2 (EXT2) genes. In this study, we report the analysis of the mutational status of the EXT2 gene in tumor samples derived from a patient affected by hereditary $\mathrm{MO}$, documenting the somatic loss of the germline mutation in a giant chondrosarcoma and in a rapidly growing osteochondroma. The sequencing of all exons and exon-intron junctions of the EXT1 and EXT2 genes from blood DNA of the proband did not reveal any mutation in the EXT1 gene but did demonstrate the presence of the transition point mutation c.67C > T in the EXT2 gene, determining the introduction of a stop codon in the coding sequence $(p$. Arg23*). A mutational analysis of other members of the family and the presence of osteochondromas in the metaphysis of long bones confirmed the diagnosis of hereditary multiple osteochondromas. Direct sequencing from DNA extracted from different sites of two tumor samples (a small rapidly growing osteochondroma and a giant peripheral secondary chondrosarcoma, each located at different chondrocostal junctions) revealed the loss of the germline EXT2 mutation. Analysis of microsatellite polymorphic markers in the $11 \mathrm{p}$ region harboring the EXT2 gene did not reveal any loss of heterozygosity. This observation supports a recent model of sarcomagenesis in which osteochondroma cells bear EXT homozygous inactivation, whereas chondrosarcoma-initiating cells are EXT-expressing cells.
\end{abstract}

Keywords Hereditary multiple osteochondromas, tumor suppressor gene, chondrosarcoma, hereditary cancer

(c) 2015 Elsevier Inc. All rights reserved.

Multiple osteochondromas (MO) is an autosomal-dominant skeletal disorder characterized by the formation of multiple cartilage-capped bony protuberances or osteochondromas. $\mathrm{MO}$ is caused by mutations in either of two genes: exostosin1 (EXT1), which is located on chromosome 8q24.11-q24.13,

Received November 7, 2014; received in revised form December 19, 2014; accepted January 11, 2015.

* Corresponding author.

E-mail address: daniele.condorelli@unict.it

${ }^{1}$ Both authors contributed equally to this work, and both are considered first author. and exostosin-2 (EXT2), which is located on chromosome 11p12-p11 (1-3). However, in $5-30 \%$ of patients, no EXT1 or EXT2 mutation can be detected $(4,5)$. Most germline mutations found in the EXT genes are nonsense, frameshift, or splice-site mutations leading to a premature stop codon inactivating the gene.

According to the two-hit model (6) for classic tumor suppressor genes, both alleles of EXT would need to be inactivated for osteochondroma formation, with one allele constitutionally inactivated and the other somatically mutated. The second somatic hit is the inactivation of the remaining wild-type copy, which occurs often either by deletion of the chromosomal region that harbors the gene or 
by mitotic recombination. This results in a loss of heterozygosity $(\mathrm{LOH})$, which is detectable with polymorphic markers located in this chromosomal region. Indeed, $\mathrm{LOH}$ of EXT1 or EXT2 and loss of the wild-type allele were shown in hereditary osteochondromas, supporting the two-hit model (7-11). However, the absence of $\mathrm{LOH}$ or of biallelic inactivation of the EXT genes in a substantial proportion of osteochondromas (12-16) resulted in a longstanding controversy as to whether osteochondromas could arise through haploinsufficiency (i.e., the single functional copy of the gene does not produce enough gene product to bring about the wild-type condition, leading to osteochondromas).

Mouse experimental genetic models have provided strong evidence in favor of the role played by biallelic inactivation of EXT genes in chondromagenesis in accordance with the classic two-hit model for tumor suppressor genes $(17,18)$. Indeed, somatic biallelic inactivation of EXT1 in a fraction of chondrocytes generates osteochondromas of the long bones in these mouse models $(17,18)$. Moreover, the discovery that chondrocytes constituting such experimental osteochondromas are mixtures of mutant and wild-type cells may explain the difficulties in detecting homozygous inactivation of the EXT genes in several human studies.

The most important complication of osteochondroma is the malignant transformation into a secondary peripheral chondrosarcoma, which is estimated to occur in $1-5 \%$ of patients (19). The presence of genetic aberrations not related to EXT has been shown during chondrosarcoma progression (12,20-22), and it is assumed that additional genetic alterations (extra-hits) are necessary to progress into malignancy, although different hypotheses have been suggested regarding the mutational status of the EXT genes in the secondary chondrosarcoma-initiating cells $(16,23,24)$.

In this study, we report the analysis of the mutational status of the EXT2 gene in two tumor samples (an osteochondroma and a peripheral secondary chondrosarcoma, each located at different chondrocostal junctions) taken from a patient affected by hereditary MO. The main point of this communication is the surprising observation that the germline-mutated EXT2 allele is somatically lost in both examined tumor samples, a result with interesting implications for the mechanism of such alteration and for the possible role of functional EXT genes in chondrosarcoma progression (25).

\section{Materials and methods}

\section{Research participants}

The patient affected by multiple osteochondromas and other participants belonging to the same genealogic tree were recruited for this study. Every participant provided a written informed consent for participating in the study.

\section{DNA extraction, PCR amplification, and sequence analysis}

Genomic DNA (gDNA) was extracted from blood cells using the QIAamp DNA blood mini kit (Qiagen, Venlo, Netherlands) according to the manufacturer's instructions. The concentration and the quality of the DNA was determined using a NanoDrop ND-1000 spectrophotometer (Thermo Fisher Scientific, Waltham, MA). Approximately $200 \mathrm{ng}$ of DNA from each individual was used in the PCR reactions. The QIAamp DNA formalin-fixed, paraffin-embedded (FFPE) tissue kit (Qiagen) was used according to the manufacturer's instructions to extract gDNA from FFPE tissue.

The PCR was performed with oligonucleotide primers specific for the coding exons of the EXT1 and EXT2 genes $(24,26)$. Exon 1 of EXT1 was split into three overlapping fragments. DNA was added to a mix containing $25 \mathrm{pmol}$ of each primer (forward and reverse), PCR buffer $10 \times, 10 \mu \mathrm{M}$ dNTP, $50 \mathrm{mM}$ magnesium chloride, and 5 units of Taq polymerase, to give a total reaction volume of $50 \mu \mathrm{L}$. Thermal conditions required for the reaction were a preheating step at $95^{\circ} \mathrm{C}$ for 30 seconds, followed by the addition of Invitrogen Platinum Taq DNA polymerase (Thermo Fisher Scientific) and 35 cycles at $95^{\circ} \mathrm{C}$ for 1 minute, $62^{\circ} \mathrm{C}$ for 1 minute, and $72^{\circ} \mathrm{C}$ for 1 minute. All PCR-product sizes were confirmed by an Agilent 2100 Bioanalyzer (Agilent Technologies, Santa Clara, CA). The PCR products were purified and $10 \mathrm{ng}$ of each PCR product was sequenced on an ABI Prism 310 Genetic Analyzer (Thermo Fisher Scientific) using the Applied Biosystems BigDye terminator cycle sequencing ready reaction kit (Thermo Fisher Scientific) as previously described (27).

\section{Pyrosequencing method}

Human genomic DNA (10 ng), extracted as previously described from blood or FFPE tissues, was PCR amplified using conditions optimized for pyrosequencing analysis (PyroMark PCR Kit, catalogue number [cat. no.] 978703; Qiagen). PCR was performed with oligonucleotide primers specific for pyrosequencing (PyroMark custom assay, cat. no. 978776; Qiagen). DNA was added to a mix containing $0.2 \mu \mathrm{M}$ of each primer (forward and reverse) in the PyroMark PCR MasterMix (Qiagen), up to a total reaction volume of $25 \mu \mathrm{L}$. Thermal conditions required for the reaction were a preheating step at $95^{\circ} \mathrm{C}$ for 15 minutes; 45 cycles at $95^{\circ} \mathrm{C}$ for 30 seconds, at $60^{\circ} \mathrm{C}$ for 30 seconds, and at $72^{\circ} \mathrm{C}$ for 30 seconds; and a final extension at $72^{\circ} \mathrm{C}$ for $10 \mathrm{mi}-$ nutes. PCR-product sizes were confirmed by $1.2 \%$ agarose gel electrophoresis. The Qiagen protocol was used for immobilization to Sepharose beads: PyroMark Q24 advanced reagent, cat. no. 970902 (Qiagen) and Streptavidin Sepharose High Performance (cat. no. 17-5113-01, GE Healthcare, Chalfont St. Giles, UK), using $10 \mu \mathrm{L}$ of PCR product. After immobilization, samples were incubated with PyroMark annealing buffer (cat. no. 979009; Qiagen) and sequencing primer and run on the PyroMark Q24.

\section{$11 p$ LOH analysis by microsatellite analysis}

Polymorphic microsatellites located in the EXT2 locus (D11S903) or surrounding it in the $11 \mathrm{p}$ chromosome (D11S1355, D11S1313, D11S1319) were analyzed by PCR amplification and measurement of their sizes on the $A B I$ Prism 310 Genetic Analyzer. 


\section{Results}

\section{Clinical case description}

In May 2006, patient II-1, a 22-year-old man, was examined by one of the authors (F.P.C.) because of a slow-growing, painless mass on the anterior chest wall (Figure 1A). Results of a CT scan and MRI showed a well-circumscribed, $18 \times 14 \times 15-\mathrm{cm}$, expansile mass with amorphous calcifications inside. Intralesional radiodensities consisted of calcified cartilage that manifested as popcorn-like speckled foci. The tumor arose from the right costochondral junction of the seventh rib, with involvement of the fifth, sixth, and eighth ribs as well as partial diaphragmatic and sternal involvement. The mass extrinsically compressed the liver and had minimal contact with the right lung (Figure 1B). A preoperative biopsy was performed, and the diagnosis of low grade chondrosarcoma was made. The patient presented with multiple osteochondromas, providing proof of the multicentricity of the disease (an X-ray image of osteochondromas of the left arm is shown in Figure $1 \mathrm{C}$ ). The presence of a small osteochondroma $(8 \mathrm{~mm})$, originating from the inner side of the anterior arc of the left third rib, was also detected. In July 2006, the patient underwent a surgical resection of the giant thoracic chondrosarcoma, and the postoperative histopathological analysis confirmed a completely resected low grade chondrosarcoma (Figure 1D, sample T1). The tumor's weight was $5,400 \mathrm{~g}$.

The patient was discharged with a strict follow-up program, including a scheduled CT scan every 6 months, because of the presence of other osteochondromas. In August 2007, a CT scan of the chest revealed that the painless $8 \mathrm{~mm}$ osteochondroma, which originated from the inner side of the anterior arc of the left third rib, had increased in size to $1.5 \mathrm{~cm}$ (Figure 1E). In September 2007, the patient underwent rib resection. Histopathology confirmed a completely resected osteochondroma (sample T2). No local recurrences have been observed at 6 years postthoracoabdominal excision and 5 years after left rib resection. Other osteochondromas are stable to date.

\section{Mutational analysis of the EXT genes in blood and tumor DNA}

The sequencing of all exons and exon-intron junctions of the EXT1 and EXT2 genes from blood DNA of patient II-1 did not reveal any mutation in the EXT1 gene but did demonstrate the presence of the transition point mutation c.67C $>\mathrm{T}$ (counting from start site in NM_207122.1) in the EXT2gene, determining the introduction of a stop codon in the coding sequence (p.Arg23*) (Figure 2A). An analysis of DNA from other family members revealed the presence of the same mutation in the father (I-1), in the paternal uncle (I-2), and in one first cousin (II-2) of the proband (Figure 2B). None of the affected relatives developed chondrosarcomas, although the presence of multiple osteochondromas was documented radiologically.

Direct sequencing of the EXT2 gene by the classic chaintermination method did not reveal the presence of the mutation p.Arg23* in DNA extracted from sample T1 (secondary peripheral chondrosarcoma) nor T2 (osteochondroma) obtained from patient II-1 (Figure 2A). The analyses were repeated in two different sites for each tumor sample.

We repeated the sequencing reactions with a more sensitive and quantitative method (pyrosequencing). With this method, we could observe the presence of the mutation c.67C > T in T1 and T2 tumor samples. However, the normal

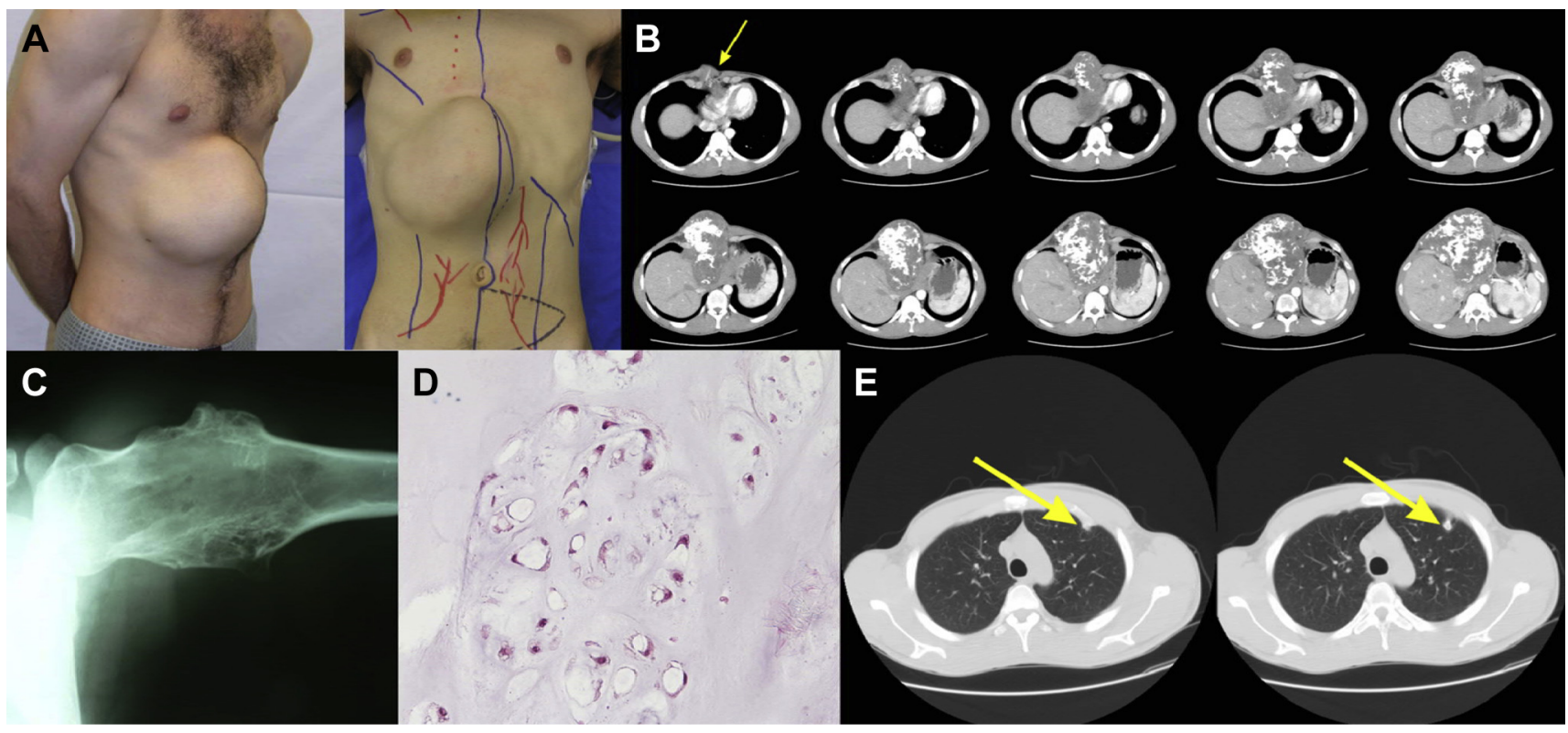

Figure 1 (A) Lateral and frontal views of the mass on the right lower anterior chest wall. (B) Results of a CT scan of the chondrosarcoma shows a large inhomogeneous mass containing scattered calcified areas. Yellow arrows show evidence of a radiological stalk. (C) X-ray images show multiple osteochondromas leading to a deformity of the left arm. (D) A histopathological image of chondrosarcoma of the chest wall after the surgical resection of the mass (T1 sample). (E) Results of a CT scan show exostoses of the inner side of the anterior arc of the left third rib (T2 sample). (Color versions of these illustrations are available on the journal's website at www.cancergeneticsjournal.org.) 


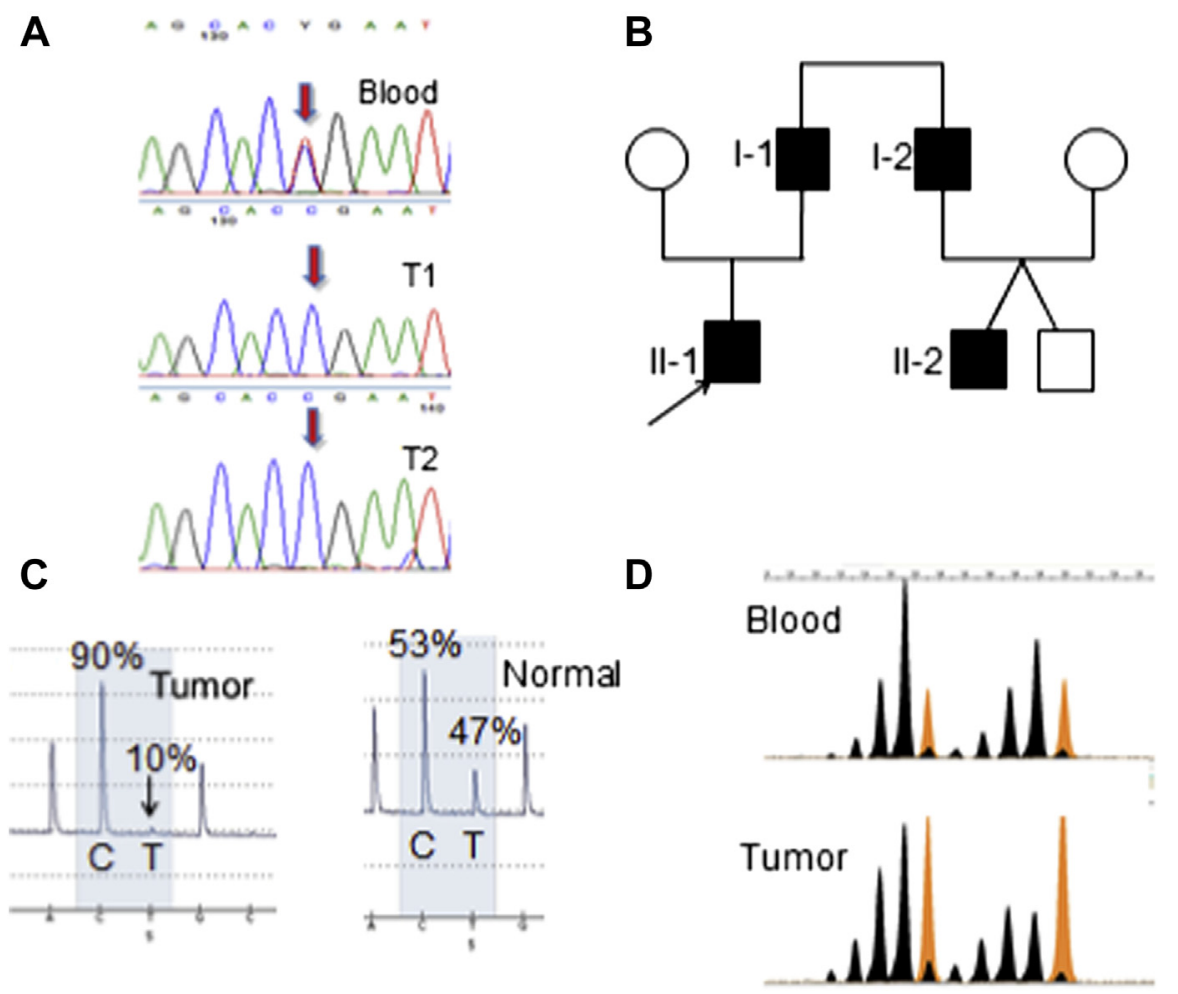

Figure 2 (A) Sequencing electropherograms show the presence of the mutation c.67 C > T in the EXT2 gene in blood DNA from patient II-1 and the absence of the mutation in tumors T1 and T2 from the same patient. (B) The family tree of patient II-1. Black symbols indicate the presence of the mutation. (C) Pyrograms show the mutation c.67 C > T in sample T2 (Tumor) and in normal chondrocostal tissue present in the same paraffin-embedded block (Normal). The percentage above the corresponding peak indicates the allele frequency. (D) An electropherogram shows PCR amplification of microsatellite D11S903 at the EXT2 locus, in blood and tumor T1 (black peaks). (Size standards are depicted as orange peaks). Similar results have been obtained in tumor T2. (Color versions of these illustrations are available on the journal's website at www.cancergeneticsjournal.org.)

C allele was present in the vast majority of the DNA molecules (see Figure 2C for sample T2 with $90 \%$ C and $10 \%$ mutated $\mathrm{T}$ alleles). DNA extracted from tissue slices of the same paraffin block that contained normal chondrocostal tissue showed the mutation with an allele frequency of $53 \%$ for $\mathrm{C}$ and $47 \%$ for $\mathrm{T}$, as expected for a germline heterozygous mutation. Analysis by pyrosequencing of DNA extracted from blood of II-1 and I-1 confirmed the presence of the mutation c.67C $>\mathrm{T}$ with the expected allele frequency for the heterozygous mutation.

\section{$11 p$ Loss of heterozygosity (LOH) analysis}

Analysis of polymorphic microsatellite markers (see Materials and methods) surrounding the EXT2 gene, located at 11p12p11, excluded the presence of an LOH region in this chromosomal region. Representative results for the microsatellite D11S903, contained in the EXT2 locus, are shown in Figure 2D.

\section{Discussion}

In this report, we document that the germline mutation of the EXT2 gene, causally linked to hereditary $M O(5,26,28)$, is somatically lost during tumorigenesis in two different tumoral samples derived from the same member of an affected family.
In the classic two-hit model (6) of hereditary tumorigenesis, the second somatic event is usually responsible for the loss of the wild-type allele, whereas in the case here reported, we observed the loss of the mutated allele and the retention of the wild-type one. Such a condition could be referred to as an "isoallelic two-hit model" to highlight that the same allele is affected twice, as opposed to the classic "alloallelic two-hit hypothesis" that predicts the different alleles are affected by the two hits (Figure 3).

Interestingly, a similar observation was reported in 1997 for the EXT1 gene, the other gene responsible for $\mathrm{MO}$. Hecht et al. (29) analyzed a chondrosarcoma derived from a patient bearing a constitutional heterozygous EXT1 mutation (a single-base insertion resulting in a frameshift and premature stop codon) and found that the tumor underwent $\mathrm{LOH}$ for chromosome 8q24.1, retaining the wild-type EXT1 allele and losing the mutated one. Indeed, a partial or complete loss of the mutation-bearing chromosome can be considered a likely mechanism for the loss of the mutated allele. It has been repeatedly reported $(7,9,12,21)$ that, in chondrosarcomas, the majority of the autosomes, including chromosome 11, frequently displayed $\mathrm{LOH}$ as a result of chromosome loss (deletion-LOH) and, in some cases, subsequent duplication of the remaining homologue gives rise to acquired uniparental disomy or copy-neutral LOH (CN-LOH) (22). Segmental CN$\mathrm{LOH}$ can also take place in a single step: for instance, through a mitotic recombination. In such case, a biallelic wild- 
Isoallelic Two-Hit

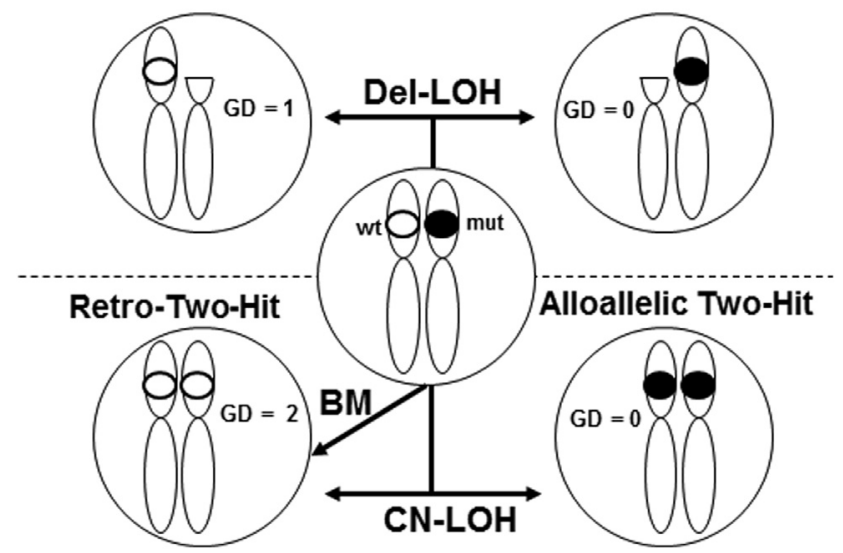

Figure 3 A schematic representation of a cell bearing a heterozygous inactivating mutation (mutated (mut) allele and wild-type (wt) allele) undergoing a second somatic hit in the wt allele (alloallelic two-hit or classic two-hit model) or in the mut allele (isoallelic two-hit or retro-two-hit). Abbreviations: Del-LOH: $\mathrm{LOH}$ due to deletion of a chromosomal region or an entire chromosome; BM: back-mutation; GD: gene dosage.

type condition $+/+$ would appear in a tumoral lineage in the context of a heterozygous organism +/-. Such somatic reversion of an inherited mutation in the context of a tumor can be referred to as a "retro two-hit model" (Figure 3). However, in the present case, the analysis of polymorphic microsatellite markers did not reveal any type of $\mathrm{LOH}$ in the chromosomal region harboring the EXT2 gene. Other mechanisms may be responsible for the observed somatic loss of the inherited mutation, such as a small focal deletion larger than the sequenced region but not involving the polymorphic markers, or a true back mutation. Indeed, examples of somatic back mutations and revertant somatic mosaicism have been reported in genetic diseases $(30,31)$. Whatever the mechanism of the loss of the mutated allele (focal CN-LOH, focal deletion, or true back-mutation) the fact that most cells in the tumor samples show a normal $\mathrm{C}$ allele suggests that the predominating cell type in chondrosarcoma (T1) or in rapidly growing osteochondromas (T2) did not originate from cells bearing a biallelically inactivated EXT2 gene.

The importance of our observation mainly relies on its impact on some aspects of the present conception of hereditary tumorigenesis. Indeed, inactivating mutations of the EXT2 gene are causally linked to the hereditary susceptibility to osteochondromas development and, in this sense, the EXT2 mutations can be considered driver mutations for osteochondromas. As reported in the introduction, either haploinsufficency or a two-hit mechanism can be invoked to explain the role of the EXT2 gene in the genesis of osteochondromas (11). However, the molecular cytogenetic characterization of human tumors and recent genetic mouse models support a two-hit mechanism, leading to biallelic inactivation of the EXT genes, as a necessary step for chondromagenesis $(17,18,32)$. Moreover, in such experimental osteochondromas, wild-type cells with functional EXT genes were being integrated into the mutated cartilaginous tumor tissue, thus producing a genetic mosaic. On the basis of such a hypothesis, osteochondromas of patient II-1 should contain a mixture of cells with different EXT2 genotypes: a population of cells $+/$ - bearing a monoallelic EXT2 inactivation because of the first hereditary hit, and a population of cells -/- bearing a biallelic inactivation of the EXT2gene generated by the second somatic hit. Therefore, the cellular and molecular events that lead to the transformation of osteochondromas in secondary chondrosarcoma should take place in the context of this genetic mosaic. The current multistep genetic model for secondary peripheral chondrosarcoma formation assumes that osteochondroma cells with homozygous inactivation of EXT1 or EXT2 are the origin of chondrosarcoma-initiating cells that acquire one or more additional genetic alterations to progress into malignancy. However, it has been suggested (25) that it is unlikely that such cells (-/-) can generate a cell population bearing the EXT2 wild-type allele. Indeed, the presence of a vast cell population bearing only the wild-type EXT2 allele in a peripheral secondary chondrosarcoma sample (as observed in the T1 sample of this study) suggests that chondrosarcomainitiating cells might be the constitutional +/- cells, and not the double-hit -/- ones. Moreover, in this study we detected similar findings in a rapidly growing osteochondroma (T2 sample), which probably represents an early stage of progression toward a peripheral secondary chondrosarcoma.

Our results are in agreement with recent data by de Andrea et al. (25) that challenged the notion that osteochondroma cells with homozygous inactivation of EXT1 or EXT2 are the cellular origin of secondary peripheral chondrosarcomas. By immunohistochemical analysis, these researchers confirmed the presence of cells with dysfunctional EXT1 in sporadic and hereditary osteochondromas and showed cells with functional EXT1 in secondary peripheral chondrosarcomas. Homozygous inactivation of the EXT1 locus in sporadic secondary peripheral chondrosarcoma $(2 / 17$ cases, $12 \%)$ is much less frequently detected than expected based on the assumption that it originates in a sporadic osteochondroma, in which homozygous inactivation of EXT1 is found in $80 \%$ of cases $(11,32)$. These results point to a model of sarcomagenesis in which the osteochondroma cells with homozygous inactivation of EXT create a niche (a permissive microenvironment), which enables cells with functional EXT to acquire other genetic changes that give rise to secondary peripheral chondrosarcoma (25).

The results of this study, considered in light of other recent investigations $(23,25)$, support the idea that osteochondromas and their associated secondary peripheral chondrosarcomas have different initiating cells. However, the conclusive claim that chondrosarcoma arises predominantly from cells with a normal EXT gene will require the accurate molecular characterization of a large collection of tumor samples deriving from well-characterized families affected by this rare genetic disease.

\section{References}

1. Ahn J, Lüdecke HJ, Lindow $S$, et al. Cloning of the putative tumour suppressor gene for hereditary multiple exostoses (EXT1). Nat Genet 1995;11:137-143.

2. Stickens D, Clines G, Burbee D, et al. The EXT2 multiple exostoses gene defines a family of putative tumour suppressor genes. Nat Genet 1996;14:25-32. 
3. Wuyts W, Van Hul W, Wauters J, et al. Positional cloning of a gene involved in hereditary multiple exostoses. Hum Mol Genet 1996;5:1547-1557.

4. Jennes I, Entius MM, Van Hul E, et al. Mutation screening of EXT1 and EXT2 by denaturing high-performance liquid chromatography, direct sequencing analysis, fluorescence in situ hybridization, and a new multiplex ligation-dependent probe amplification probe set in patients with multiple osteochondromas. J Mol Diagn 2008;10:85-92.

5. Jennes I, Pedrini E, Zuntini M, et al. Multiple osteochondromas: mutation update and description of the multiple osteochondromas mutation database (MOdb). Hum Mutat 2009;30:1620-1627.

6. Knudson AG Jr. Mutation and cancer: Statistical study of retinoblastoma. Proc Natl Acad Sci U S A 1971;68:820-823.

7. Hecht JT, Hogue D, Strong LC, et al. Hereditary multiple exostosis and chondrosarcoma: linkage to chromosome II and loss of heterozygosity for EXT-linked markers on chromosomes II and 8. Am J Hum Genet 1995;56:1125-1131.

8. Bridge JA, Nelson M, Orndal C, et al. Clonal karyotypic abnormalities of the hereditary multiple exostoses chromosomal loci 8q24.1 (EXT1) and 11p11-12 (EXT2) in patients with sporadic and hereditary osteochondromas. Cancer 1998;82:1657-1663.

9. Bovée JV, Cleton-Jansen AM, Wuyts W, et al. EXT-mutation analysis and loss of heterozygosity in sporadic and hereditary osteochondromas and secondary chondrosarcomas. Am J Hum Genet 1999;65:689-698.

10. Feely MG, Boehm AK, Bridge RS, et al. Cytogenetic and molecular cytogenetic evidence of recurrent 8q24.1 loss in osteochondroma. Cancer Genet Cytogenet 2002;137:102-107.

11. Reijnders CM, Waaijer CJ, Hamilton A, et al. No haploinsufficiency but loss of heterozygosity for EXT in multiple osteochondromas. Am J Pathol 2010;177:1946-1957.

12. Bovée JVMG, Cleton-Jansen AM, Kuipers-Dijkshoorn N, et al. Loss of heterozygosity and DNA ploidy point to a diverging genetic mechanism in the origin of peripheral and central chondrosarcoma. Genes Chromosomes Cancer 1999;26:237-246.

13. Hecht JT, Hall CR, Snuggs M, et al. Heparan sulfate abnormalities in exostosis growth plates. Bone 2002;31:199-204.

14. Hall CR, Cole WG, Haynes R, et al. Reevaluation of a genetic model for the development of exostosis in hereditary multiple exostosis. Am J Med Genet 2002;112:1-5.

15. Benoist-Lasselin C, de Margerie E, Gibbs L, et al. Defective chondrocyte proliferation and differentiation in osteochondromas of MHE patients. Bone 2006;39:17-26.

16. Zuntini M, Pedrini E, Parra A, et al. Genetic models of osteochondroma onset and neoplastic progression: evidence for mechanisms alternative to EXT genes inactivation. Oncogene 2010;29:3827-3834.

17. Jones KB, Piombo V, Searby $C$, et al. A mouse model of osteochondromagenesis from clonal inactivation of Ext1 in chondrocytes. Proc Natl Acad Sci U S A 2010;107:2054-2059.

18. Matsumoto K, Irie F, Mackem S, et al. A mouse model of chondrocyte-specific somatic mutation reveals a role for Ext1 loss of heterozygosity in multiple hereditary exostoses. Proc Natl Acad Sci U S A 2010;107:10932-10937.

19. Wicklund CL, Pauli RM, Johnston D, et al. Natural history study of hereditary multiple exostoses. Am J Med Genet 1995;55: 43-46.

20. Lee FY, Zawadsky M, Parisien M, et al. Novel translocation (9; 12)(q22;q24) in secondary chondrosarcoma arising from hereditary multiple exostosis. Cancer Genet Cytogenet 2002;132: 68-70.

21. Bovée JV, van Royen M, Bardoel AF, et al. Near-haploidy and subsequent polyploidization characterize the progression of peripheral chondrosarcoma. Am J Pathol 2000;157: 1587-1595.

22. Olsson L, Paulsson K, Bovée JV, et al. Clonal evolution through loss of chromosomes and subsequent polyploidization in chondrosarcoma. PLoS One 2011;6:e24977.

23. de Andrea CE, Wiweger MI, Bovée JV, et al. Peripheral chondrosarcoma progression is associated with increased type $\mathrm{X}$ collagen and vascularisation. Virchows Arch 2012;460:95-102.

24. Wuyts W, Van Hul W. Molecular basis of multiple exostoses: mutations in the EXT1 and EXT2 genes. Hum Mutat 2000;15: 220-227.

25. de Andrea CE, Reijnders CM, Kroon HM, et al. Secondary peripheral chondrosarcoma evolving from osteochondroma as a result of outgrowth of cells with functional EXT. Oncogene 2012; 31:1095-1104.

26. Vink GR, White SJ, Gabelic S, et al. Mutation screening of EXT1 and EXT2 by direct sequence analysis and MLPA in patients with multiple osteochondromas: splice site mutations and exonic deletions account for more than half of the mutations. Eur J Hum Genet 2005;13:470-474.

27. Barresi V, Romano A, Musso N, et al. F. Broad copy neutralloss of heterozygosity regions and rare recurring copy number abnormalities in normal karyotype-acute myeloid leukemia genomes. Genes Chromosomes Cancer 2010;49: 1014-1023.

28. Signori E, Massi E, Matera MG, Poscente M, et al. A combined analytical approach reveals novel EXT1/2 gene mutations in a large cohort of Italian multiple osteochondromas patients. Genes Chromosomes Cancer 2007;46:470-477.

29. Hecht JT, Hogue D, Wang Y, et al. Hereditary multiple exostoses (EXT): mutational studies of familial EXT1 cases and EXT-associated malignancies. Am J Hum Genet 1997;60: 80-86.

30. Ellis NA, Ciocci S, German J. Back mutation can produce phenotype reversion in Bloom syndrome somatic cells. Hum Genet 2001;108:167-173.

31. Pasmooij AM, Pas HH, Deviaene FC, et al. Multiple correcting COL17A1 mutations in patients with revertant mosaicism of epidermolysis bullosa. Am J Hum Genet 2005;77:727-740.

32. Hameetman L, Szuhai K, Yavas A, et al. The role of EXT1 in nonhereditary osteochondroma: identification of homozygous deletions. J Natl Cancer Inst 2007;99:396-406. 